Revue de droit comparé du travail et de la sécurité sociale

$3 \mid 2020$

La Directive 2019/1158 du 20 juin 2019 concernant l'équilibre entre vie personnelle et vie privée des parents et des aidants

\title{
Emploi occasionnel et normes du travail en Australie
}

Shae McCrystal

\section{OpenEdition}

Journals

Édition électronique

URL : https://journals.openedition.org/rdctss/1032

DOI : $10.4000 /$ rdctss. 1032

ISSN : 2262-9815

Éditeur

Centre de droit comparé du travail et de la sécurité sociale

Édition imprimée

Date de publication : 1 novembre 2020

Pagination : 188-191

ISSN : 2117-4350

Référence électronique

Shae McCrystal, «Emploi occasionnel et normes du travail en Australie », Revue de droit comparé du travail et de la sécurité sociale [En ligne], 3 | 2020, mis en ligne le 01 novembre 2021, consulté le 11 novembre 2021. URL : http://journals.openedition.org/rdctss/1032 ; DOI : https://doi.org/10.4000/ rdctss. 1032

\section{(c) (i) $\odot$}

Revue de droit comparé du travail et de la sécurité sociale est mise à disposition selon les termes de la Licence Creative Commons Attribution - Pas d'Utilisation Commerciale - Pas de Modification 4.0 International. 


\title{
SHAE MCCRYSTAL
}

\author{
UNIVERSITÉ DE SYDNEY
}

\section{EMPLOI OCCASIONNEL ET NORMES DU TRAVAIL EN AUSTRALIE}

La montée du phénomène des contrats "zéro heure », et d'autres formes d'emploi occasionnel intermittent existant dans la gig economy, a mis en évidence le besoin pressant d'une législation protégeant davantage les travailleurs précaires du monde entier. Des solutions réglementaires doivent être trouvées, pour garantir aux travailleurs un traitement digne et équitable, et s'assurer qu'on ne les considère pas comme des produits jetables, à choisir sur des « rayonnages » virtuels à la convenance des entreprises.

En Australie, la réglementation du travail protège traditionnellement les « personnels occasionnels ». Cependant, deux décisions récentes de la Cour fédérale d'Australie ont mis en évidence des problèmes systémiques dans l'approche australienne. La réglementation des travailleurs occasionnels en Australie et les complexités du régime font l'objet de cette mise à jour. L'une des particularités de la réglementation australienne en matière de travail occasionnel est la manière de déterminer le statut du travailleur occasionnel. Une personne peut être considérée comme un travailleur occasionnel par une forme de réglementation mais pas par une autre, selon la définition que l'on donne au terme « occasionnel ». II existe des différences marquées entre la Common Law, le droit statutaire et les instruments de droit industriel tels que les conventions et les accords d'entreprise.

En Common Law, les questions de savoir si une personne est un salarié et la nature de son emploi relèvent de questions de droit, déterminées par le contrat en vertu duquel la personne est engagée. Comme dans de nombreux systèmes à l'étranger, l'existence d'un contrat de travail est déterminée par une série de facteurs, notamment le degré de contrôle exercé par l'employeur, l'intégration du travailleur dans l'entreprise de l'employeur et la mesure dans laquelle le travailleur travaille pour son propre compte ${ }^{1}$. Lorsqu'un contrat de travail existe, le fait qu'il s'agit d'un contrat à durée déterminée, à durée indéterminée ou occasionnel dépendra des conditions d'exécution du travail. Dans un contrat occasionnel, on trouve des indices de "schémas de travail irréguliers, d'incertitude, d'intermittence du travail et d'imprévisibilité $»^{2}$.

Une fois engagés dans le cadre d'un contrat de travail, les droits des salariés seront essentiellement issus de la législation et des instruments de droit industriel. En Australie, la plupart des travailleurs sont couverts par la loi de 2009 sur le travail équitable (Fair Work Act ou FW Act, Commonwealth) qui prévoit des normes minimales en matière d'emploi concernant, entre autres, les droits aux congés, les heures de travail et les licenciements abusifs. Le FW Act prévoit également la création de conventions collectives modernes, qui fixent des normes du travail plus détaillées applicables par secteur d'activité ou par profession, et des accords d'entreprise négociés entre un employeur et ses salariés et s'appliquant à l'échelle de l'entreprise.

1 Hollis v Vabu Pty Ltd (2001) 207 CLR 21, voir également A. Stewart et S. McCrystal, "Labour Regulation and the Great Divide: Do New Business Models Require a New Category of Worker?", Australian Journal of Labour Law, 2019, vol. 32, p. 4.

2 Workpac Pty Ltd $v$ Skene [2018] FCAFC 131 at [172]. 
La plupart des conventions collectives et des accords comprennent une définition des différents types d'emploi, qui s'appliquent uniquement aux fins de l'instrument concerné. Une définition courante est qu'un salarié occasionnel est un travailleur " engagé et rémunéré en tant que travailleur occasionnel». En d'autres termes, quelle que soit la nature de l'engagement, si le contrat stipule qu'un travailleur est un salarié occasionnel et qu'il est rémunéré comme un salarié occasionnel, le travailleur est un salarié occasionnel et ne bénéficie pas d'un emploi permanent au regard des droits conférés par l'instrument en question. Cela permet d'engager des travailleurs de façon informelle, sachant que leurs horaires ne sont pas garantis et que les droits tels que les congés ne sont pas accordés, mais qu'ils reçoivent en contrepartie un salaire horaire plus élevé, pour compenser la précarité et l'irrégularité persistantes. Cette prime de précarité est généralement fixée à $25 \%$ du salaire horaire de base d'un salarié en contrat à durée indéterminée. La possibilité d'avoir recours à cette formule a conduit en Australie à un phénomène connu sous le nom de "travail occasionnel permanent » dans lequel un travailleur est engagé et rémunéré comme un salarié occasionnel, mais ses horaires de travail et la fréquence de ses missions font qu'il travaille en réalité à temps plein - ou à temps partiel - et pour une durée indéterminée.

Cette dichotomie entre la Common Law et la définition de l'emploi occasionnel dans les conventions et accords d'entreprise est reconnue depuis longtemps. Or, dans un contexte où la plupart des conditions d'engagement d'un travailleur se trouvent non pas dans son contrat de travail, mais dans une convention collective ou un accord d'entreprise, cela n'a pas posé de difficultés significatives.

Cependant, comme vu ci-avant, le FW Act comporte certains droits en matière d'emploi. Le FW Act ne contient aucune définition du salarié « occasionnel ». Les travailleurs occasionnels (sans définition) sont exclus de certains droits, alors que les salariés occasionnels permanents bénéficient d'autres droits. Par exemple, en vertu de la loi sur le licenciement abusif, un salarié « occasionnel » peut contester l'équité de son licenciement s'il a été engagé par le même employeur pendant 12 mois ou plus, de façon régulière et systématique, et s'attendait à bénéficier d'un emploi continu ${ }^{3}$. Un travailleur considéré comme occasionnel en vertu d'une convention ou d'un accord, qui est rémunéré comme un travailleur occasionnel, sera protégé contre le licenciement s'il est effectivement un travailleur permanent ${ }^{4}$. II existe donc une incohérence entre d'une part, le salaire et les conditions de travail du salarié et, d'autre part, la protection conférée par le FW Act contre le licenciement. Dans la pratique, cette dichotomie agit comme une protection pour les « occasionnels à long terme » et leur donne la possibilité de transformer leur emploi en poste permanent.

Néanmoins, le problème qui s'est posé concerne la définition du salarié occasionnel aux fins des droits en matière de congés annuels et de congés pour raisons personnelles (maladie et congé d'aidant). Le droit à des congés annuels conféré par le FW Act est défini dans l'article 87 qui accorde 4 semaines (ou 20 jours) de congé annuel aux salariés à temps plein couverts par la Loi, le prorata pour les salariés à temps partiel, et 5 semaines de congé pour les travailleurs à quarts. Cependant, les droits énoncés à l'article 87 s'appliquent aux "salariés, autres que les travailleurs occasionnels " ${ }^{5}$. Il en va de même s'agissant du droit à 10 jours par an de congé pour raison personnelle, accordé par la même loi ${ }^{6}$. Le terme

3 FW Act s 384.

4 Voir par exemple Ponce v DJT Staff Management Services [2010] FWA 2078.

5 FW Act s 86.

6 FW Act ss 95, 96. 
" occasionnel » n'est pas défini et aucune autre approche des travailleurs occasionnels à long terme dans la loi ne s'applique.

Aux fins du droit aux congés annuels et personnels en vertu du FW Act, quelle définition prévaut: celle contenue dans toute réglementation ou convention pertinente, ou la Common Law ? La réponse est d'une importance cruciale, étant donné la prévalence en Australie de ce que l'on appelle les « occasionnels permanents ", engagés et rémunérés comme des occasionnels en vertu de conventions et d'accords d'entreprise, mais traités dans la pratique comme des salariés permanents. Beaucoup de ces travailleurs acceptent un tel statut en raison de la prime de précarité de $25 \%$ qui est conçue pour compenser l'insécurité, le travail instable et, en théorie, la perte des droits conférés par le FW Act, y compris les droits aux congés annuels et personnels. Cette prime de précarité a pour effet d'augmenter le salaire net hebdomadaire d'un salarié occasionnel au détriment des autres droits en matière d'emploi. Pour les travailleurs occupant un emploi précaire ou faiblement rémunéré, en particulier ceux qui sont embauchés à la semaine, c'est un compromis qui a du sens puisqu'il permet d'obtenir une augmentation du salaire hebdomadaire. Cependant, cela devient un problème lorsque ces travailleurs veulent s'absenter du travail pour se reposer, se détendre ou en cas de maladie, puisqu'ils doivent alors prendre un congé sans solde.

Le problème a fait l'objet de deux affaires récentes devant l'assemblée plénière de la Cour fédérale d'Australie, portant essentiellement sur le même scénario factuel, Workpac v Skene (ou «Skene ») $)^{7}$ et Workpac v Rossato (ou « Rossato ») ${ }^{8}$. Ces affaires concernaient des travailleurs de l'industrie minière où le recours à l'emploi « occasionnel permanent » est courant. Les deux cas concernaient des travailleurs engagés sur une période de plusieurs années, travaillant par quarts de 12 heures sur des missions de 7 ou 14 jours (7/14 jours de travail et $7 / 14$ jours de congé), selon un arrangement dans lequel leur tableau de service était établi annuellement. Dans chaque cas, les salariés avaient été embauchés en vertu d'un contrat de travail qui stipulait expressément que l'engagement concernait un " salarié occasionnel » et que selon l'accord d'entreprise en place, un salarié occasionnel était un salarié engagé et rémunéré comme tel. Chaque travailleur était payé selon un taux horaire fixe pour les heures travaillées, mais ne recevait aucune indemnité de congé annuel ou de congé personnel.

Si les points de droit soulevés par ces affaires étaient légèrement différents, la Cour devait essentiellement déterminer comment définir le terme " occasionnel » aux fins de la non-éligibilité au droit à des congés. Autrement dit, le terme " occasionnel » utilisé dans le FW Act dans le contexte des dispositions sur les congés devait-il être interprété par référence à la Common Law, ou à la définition de la convention collective de l'industrie minière ou de l'accord collectif d'entreprise applicable sur le site.

Les salariés ont fait valoir que le FW Act devait être interprété en fonction de la Common Law, en vertu de laquelle il semblait qu'ils étaient liés par des contrats à durée indéterminée. L'employeur a fait valoir que les textes de droit industriel devaient prévaloir, étant donné la relation entre le FW Act, les conventions et accords collectifs de travail, et compte tenu de la prime de précarité reçue pour indemniser les travailleurs ayant perdu des droits tels que les congés annuels. 


\section{Australie}

Pour comprendre la position des employeurs, il faut rappeler qu'avant 2006, les droits aux congés annuels et autres figuraient dans les textes de droit industriel, et que le développement de la prime de précarité s'est produit dans ce contexte, où les travailleurs occasionnels "engagés et payés comme tels" se sont vu refuser le droit au congé par le même instrument. Lorsque les droits ont été transférés dans le droit statutaire et que les « occasionnels » en ont été exclus, il semble qu'on ait simplement supposé que la situation préexistante se poursuivrait. Cependant, la difficulté de cette approche est qu'elle fonctionne à rebours ; les instruments législatifs créés en vertu d'un statut étant utilisés pour donner un contenu au sens du statut. Ceci est particulièrement problématique quand il n'y a pas de définition unique du terme occasionnel dans ces textes réglementaires.

En fin de compte, il a été jugé que la définition du caractère occasionnel donnée par le FW Act n'était pas subordonnée à la définition du même terme dans l'instrument de droit industriel pertinent, et que le statut des travailleurs concernés devait être déterminé en fonction du fait que le contrat de travail soit jugé, en Common Law, comme occasionnel ou permanent. Pour les travailleurs, il a été jugé que l'engagement n'était pas intermittent ou imprévisible et qu'ils n'étaient pas des salariés occasionnels aux fins du droit à un congé annuel en vertu du FW Act. II a également été constaté que le droit aux congés annuels acquis non payés ne pouvait être compensé par la prime de précarité de $25 \%$ qui avait, en théorie, été versée aux travailleurs. La décision Rossato est actuellement en appel devant la Haute Cour d'Australie.

Le problème créé par ces décisions réside dans le fait que les employeurs ayant engagé du personnel désigné comme "salarié occasionnel permanent »-parce que la convention ou la réglementation le leur permet - peuvent se retrouver confrontés à de nombreuses réclamations de la part de leurs salariés occasionnels, relatives notamment à l'absence de congés annuels ou personnels rémunérés, alors qu'ils ont versé un salaire majoré d'une prime de précarité à titre de compensation pour la perte de ces congés. Pour certains employeurs, l'ampleur de la facture portant sur ces congés non payés pourrait être écrasante.

Cependant, la décision remet également en question une caractéristique de longue date du droit du travail australien, à savoir le fait que les salariés qui ne sont pas vraiment occasionnels peuvent être traités comme tels, et se voir refuser un congé annuel ou un congé pour raison personnelles, parce qu'ils ont reçu un salaire horaire plus élevé. La difficulté, dans ce cas, est clairement illustrée par la crise de la COVID dans la mesure où certains salariés, même en étant malades, ont continué à aller travailler puisqu'ils ne recevaient pas d'indemnité en cas de maladie, et ont donc été impliqués dans la propagation du virus ${ }^{9}$.

L'indemnisation des congés de maladie, des congés pour cause de responsabilités familiales et des jours de repos et de loisirs est d'une importance fondamentale pour le bien-être mental et physique de toute communauté. Ces affaires offrent l'opportunité aux régulateurs australiens de repenser le régime réglementaire en faveur des travailleurs permanents et des travailleurs véritablement occasionnels, en Australie. Une table ronde gouvernementale examine actuellement la question; il faut espérer qu'une solution offrant à tous les travailleurs des droits appropriés en matière de congés sera préférée à une solution qui préserve le statu quo préexistant.

9 Voir par exemple https://www.sbs.com.au/news/the-feed/what-does-the-spread-of-covid-19mean-for-casual-workers 